\title{
Investigation of edge formation during the coating process of Li-ion battery electrodes
}

\author{
Sandro Spiegel $\mathbb{D}^{\text {, }}$, Thilo Heckmann, Andreas Altvater, Ralf Diehm, Philip Scharfer, Wilhelm Schabel
}

Received: 21 December 2020/Revised: 31 May 2021/ Accepted: 4 June 2021

(c) The Author(s) 2021

\begin{abstract}
In this manuscript, a method to reduce superelevations of lateral edges in cross-web direction during slot die coating of shear-thinning slurries for Liion battery electrodes (LIB) was developed. Therefore, the impact of the inner slot die geometry on the edge elevations was investigated. These elevations of the coating could be almost eliminated by optimizing the flow profile at the outlet of the slot die by modification of the internal geometry. This adaption is an essential step in optimizing the coating quality of slot die coating for battery electrodes to significantly reduce coating edges and, hence, the resulting production reject during the coating step of the industrial roll-to-roll process. It was also shown that lateral edges of the coating can be influenced explicitly by process parameters such as volume flow and gap between slot die and substrate. This correlation has already been shown for other shear-thinning material systems in previous works, which is now confirmed for this material system. At the beginning, the influence of different internal geometries on the formation of the edge elevations was shown. Finally, for the shear-thinning electrode slurry used in this work, optimal dimensions of the previously determined inner geometry for the slot die outlet were
\end{abstract}

This paper was presented at the 2020 International Society of Coatings Science and Technology Conference that was held virtually September 20-23, 2020.

S. Spiegel ( $\varangle)$, T. Heckmann, A. Altvater,

R. Diehm, P. Scharfer, W. Schabel

Thin Film Technology, Karlsruhe Institute of Technology,

Kaiserstraße 12, 76131 Karlsruhe, Germany

e-mail: sandro.spiegel@kit.edu

S. Spiegel, T. Heckmann, A. Altvater, R. Diehm,

P. Scharfer, W. Schabel

Material Research Center for Energy Systems (MZE),

Karlsruhe Institute of Technology, Kaiserstraße 12, 76131

Karlsruhe, Germany found. The optimization was performed for a state-ofthe-art electrode area capacity (approximately $2.2 \mathrm{mAh} \mathrm{cm}^{-2}$ ). The results enable a significant reduction of defects and reject in the coating step of largescale production of LIB electrodes in the future, adding to a more sustainable battery production.

Keywords Slot die coating, Lithium-ion batteries, Electrode production, Edge formation, Shim geometries
Abbreviations
Li Lithium
LIB Lithium-ion battery
CMC Carboxymethyl cellulose
SBR Styrene-butadiene rubber

\section{Introduction}

In today's battery cell production, the slot die coating process in large roll-to-roll systems is state of the art. ${ }^{9}$ In the process chain, the coating step succeeds the mixing step. After the coating has been applied, the electrodes are dried in a slot-nozzle dryer, cut to size, and pressed (calendered) with a high line force to adjust the desired volumetric capacity. ${ }^{2}$ At the end of the process chain, the electrodes are post dried $^{3}$ and assembled into Li-ion cells and encased. In the battery cell production, the individual steps in the process chain influence the respective subsequent step. As a premetered coating method with a very high accuracy of the wet film height profile, slot die coating is the state of the art in large-scale production and has been scientifically investigated in many aspects. ${ }^{4}$ The influence of the cavity of a slot die on the cross-web profile 
of the wet film has been thoroughly investigated in different publications. ${ }^{5,6}$ Due to the complexity of the cavity, it should not be changed once designed. Therefore, in this study, a simpler method to influence the cross-web profile of the wet film is presented.

Besides the known coating limits of air entrainment, low-flow limit, barring, and swelling, elevations at the lateral coating edges occur in the coating step, leading to defects in subsequent process steps, and thus, to production reject. These edge elevations can superpose when the electrodes are wound into rolls, which can cause cracks in the dry electrode (see Fig. 1a). ${ }^{4,7}$ In Fig. 1, the defects resulting from edge elevations are shown.

During the calendering step, inhomogeneous stress distribution caused by superelevated edges can lead to waves and cracks in the electrode and also to local density fluctuations (see Fig. 1b). ${ }^{4,8}$ Edge elevations formed at the two-phase boundary between the fluid and free surface by a neck-in flow in cross-web direction when the fluid leaves the slot die (see Fig. 2b). ${ }^{4,9}$ This flow transports the material stored in the lateral menisci below the slot die due to conservation of mass in cross-web direction to the inside of the coating. ${ }^{4,7,9,10}$ Various mechanisms can cause the neck-in flow. In case of very low-viscous fluids, low coating speeds, as well as small wet film thicknesses $\left(h_{\text {wet }}\right)$ and large gaps $\left(h_{\mathrm{G}}\right)$, both surface tension and viscoelasticity (die swell) are dominant mechanisms., In contrast, battery slurries are highly viscous and are applied at very high coating speeds (typically up to $\left.100 \mathrm{~m} \mathrm{~min}^{-1}\right),{ }^{11}$ where viscoelasticity and surface tension influence are less dominant and are excluded as minor mechanisms. ${ }^{4,7}$ Instead, the ratio $\left(\frac{u}{\bar{u}_{\text {slurry }}}\right)$ between the web speed $u$ and the average flow velocity in the gap $\bar{u}_{\text {slurry }}$ are responsible for the formation of the neck-in flow. ${ }^{4,9,10}$ In Fig. 2, a side view of the slot die with a flow profile under the die (a) and the neck-in flow and the resulting edge elevations (b) are shown.

If the gap $h_{\mathrm{G}}$ is larger than the wet film thickness $h_{\text {wet }}$, the ratio of web speed and average flow velocity

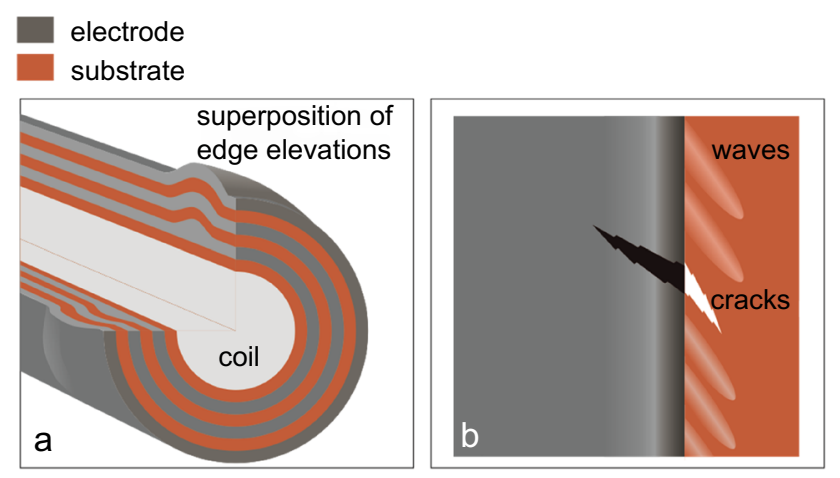

Fig. 1: Schematic illustration of superposition of edge elevations (a) (left side) and waves and cracks during the calendaring step (b) (right side) $\left(\frac{u}{\bar{u}_{\text {slury }}}\right)$ increases. Therefore, the neck-in flow also increases resulting in higher edge elevations. ${ }^{7,10}$

The resulting superelevated edges can be cut away as waste in the roll-to-roll continuous and intermittent coating process. However, not all edges can be cut off, as the electrodes must be contacted via an uncoated area of metal foil (current collectors), which can be placed on the side of the coating or between coating patches. In case of a continuously coated electrode (Fig. 3a), the current collectors must be placed at the lateral edges of the coating which makes it impossible to cut off the side edge at point (1). In Fig. 3, the cutting lines (white dashed line) for continuous and intermittent coatings are shown.

In the intermittent coating technique, the coating is applied disruptively onto the substrate to create a defined space between individual electrode patches, in which the current collectors can be placed (Fig. 3b). For intermittent coatings, the lateral edges can be cut off, but this leads to reject in the manufacturing process. ${ }^{4,12,13}$ Therefore, the lateral edges for both coating technologies must be avoided in the first place.

It is known from the literature, that the neck-in flow for high-viscous shear-thinning dispersions can be slowed down by reducing the ratio of the gap distance $h_{\mathrm{G}}$ and wet film thickness $h_{\text {wet }}$, thus, reducing the formation of edge elevations. ${ }^{7,10}$ However, the adjustment of the gap is disadvantageous for the coating of viscoelastic fluids, as it would increase the stresses in the fluid and increase the risk of barring and swelling. ${ }^{7,9}$ Swelling sets the upper process limit (maximum wet film thickness $h_{\text {wet,max }}$ ) of coating defects. In this case, the slurry leaks out of the gap at the upstream lip against the coating direction. ${ }^{14}$ If the wet film thickness $h_{\text {wet }}$ is approximately equal to or greater than the gap, barring can occur. ${ }^{14}$ This leads to periodic waves in cross-web direction and, thus, to an inhomogeneous coating. ${ }^{7}$ Air entrainment sets the lower process limit (minimum wet film thickness $h_{\text {wet,min }}$ ). In this case, air is trapped in the coating bead, resulting in air bubbles or streaks. ${ }^{7}$ The lower limit can also be set by the low-flow limit. In this case, the meniscus at the downstream lip becomes unstable, which is visible by stripes in coating direction. ${ }^{15,16} \mathrm{~A}$ close gap adjustment can also lead to further problems such as particle blocking and a higher pressure drop in the slot die. In some cases, a close enough gap cannot be adjusted due to machine-accuracy limitations. 7,11

Therefore, it is necessary to modify the neck-in flow and, thus, the coating edges independently of the gap distance $h_{\mathrm{G}}$. Han et al. have shown that the neck-in flow of Newtonian fluids can be minimized by locally reducing the outlet flow on the coating side. This reduction was achieved by suitable internal geometries of the slot die. By widening the outlet width of the die toward the outlet, the flow velocity can be reduced locally. This adjustment results in a change of the distribution of the outlet pressure in the die. It was shown that this configuration reduces edge elevations. 


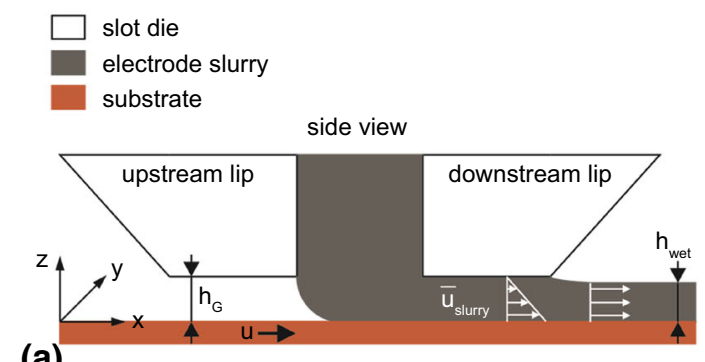

(a)

(b)

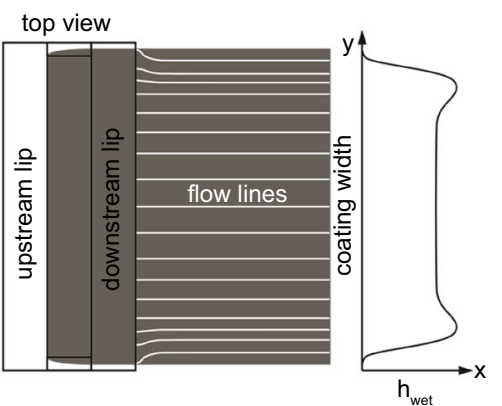

Fig. 2: Schematic illustration of the slot die (side view) with flow profile (a) (left side) and the neck-in flow with flow lines and the resulting wet film thickness cross-web profile caused by the acceleration of the flow in cross-web direction (b) (right side) $)^{4}$

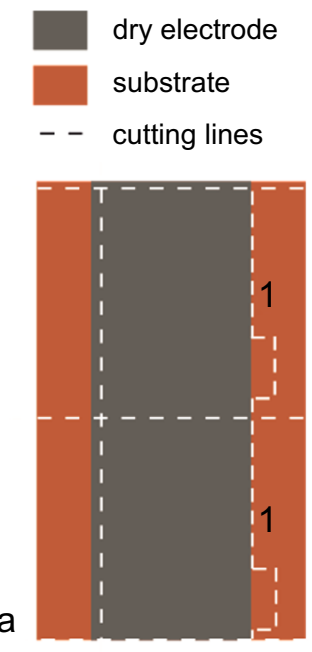

continuous

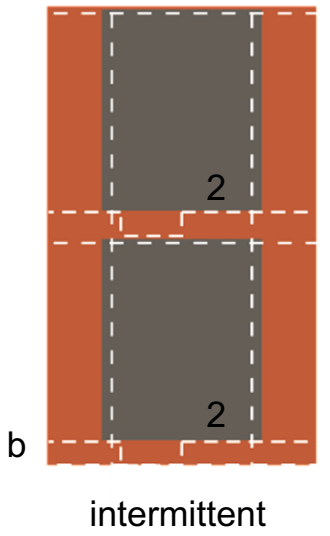

Fig. 3: Schematic drawing of the cutting lines in continuous (a) and intermittent coating (b)

A constriction of the outlet width toward the outlet has the opposite effect. Han et al. also showed that the modification of the inner geometry of the slot die can negatively affect the process window. ${ }^{17}$ An investigation for shear-thinning fluids is not yet available in the literature.

The aim of this work is to eliminate the edge elevations that occur during the coating process of $\mathrm{Li}$ ion battery electrodes by an internal configuration of the slot die. For this purpose, known influences of internal geometries on the formation of edge elevations in Newtonian slurries are transferred to shearthinning battery slurries and investigated on pilot plant scale. It is to be expected that the impact for shearthinning slurries is the same as Han et al. found for Newtonian fluids. ${ }^{17}$ As described above, the influence of the ratio of coating speed and the average flow velocity in the gap is decisive for the formation of the neck-in flow and, thus, for the formation of the edge elevations. Consequently, by reducing the local outlet flow laterally at the outlet of the slot die, the edge elevations of shear-thinning coatings can be reduced. Subsequently, the promising diverging outlet of the slot die Han et al. found is investigated by varying the geometry at the die outlet, aiming to eliminate the lateral edges. As Han et al. also determined a negative influence of the inner geometry of the slot die on the process window, operating points that are located in the stable area of the coating window must be selected for the investigation. ${ }^{17}$ In addition, known influences of process parameters on the formation of edge elevations in battery slurries are examined for the electrode slurry used.

\section{Experimental methods and materials}

\section{Formulation, mixing, and preparation}

A water-based anode slurry with $43 \%$ solids content was used for the coating experiments. The slurry was prepared with a planetary mixer (INOUE MFG., INC.) in separate mixing and kneading steps. The formulation consisted of a flake-shaped synthetic graphite (SMG-A, Hitachi Chemical Co. Ltd.), which was mixed with conductive carbon black (Super C65, Timcal) in a dry mixing step. Deionized water and a carboxymethyl cellulose (CMC) solution (Sunrose CMC MAC500LC, Nippon Paper Industries) were added in three dilution steps with kneading times of 10 , 30 , and $120 \mathrm{~min}$. In a final mixing step, a styrenebutadiene rubber (SBR) dispersion (BM-400, Zeon Corporation) was added. The composition of the solid components in the anode slurry is shown in Table 1.

For rheology investigations, the slurry was sheared using a plate-plate rotational rheometer with a $25 \mathrm{~mm}$ diameter measuring head at shear rates of 1 to $5000 \mathrm{~s}^{-1}$ at $25^{\circ} \mathrm{C}$. In Fig. 4 , the viscosity of the used electrode slurry is shown as blue dots.

The viscosity of the electrode slurry shows a shearthinning behavior. A power-law fit is valid for the investigated shear-rate range (dark blue line). The surface tension is $66 \mathrm{mN} \mathrm{m}^{-1}$. 
Table 1: Composition of the solid components of the anode slurry

Component

Graphite

Carbon black

CMC

SBR

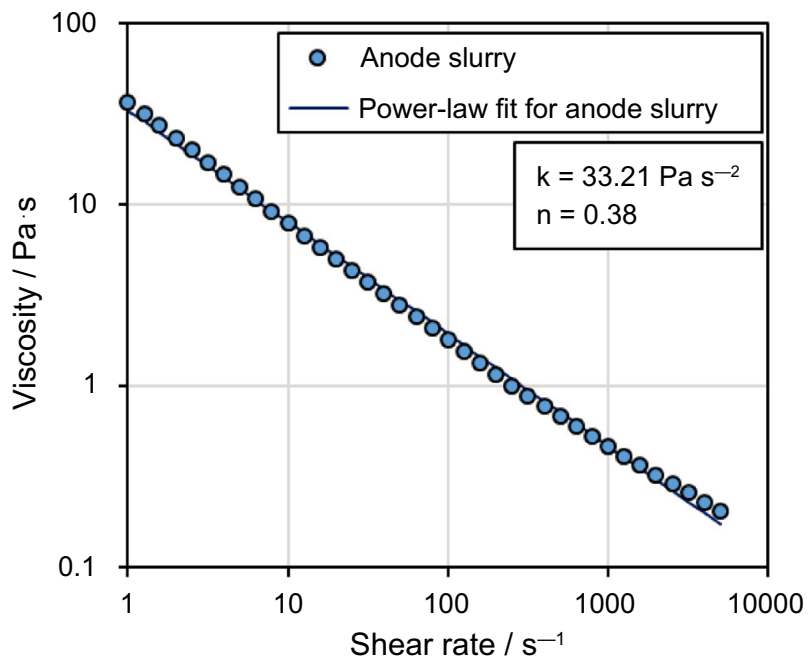

Fig. 4: Average viscosity curve of the electrode slurry as a function of the shear rate

\section{Experimental setup and process parameters}

The coating experiments were carried out on a development coater with a slot die in 8 o'clock position on a Development Coater with a high-precision steel roller (TSE Troller AG). This setup is suitable for the investigation of coatings independent of the drying step. The experimental setup is shown in Fig. 5.

Due to the high concentricity of the bearings and the roll, the experimental setup allows very precise gaps between slot die lips and high-precision roller. Coating speeds of 5 to $50 \mathrm{~m} \mathrm{~min}^{-1}$ were set for the experiments. The gap between the die and roller was set to $180 \mu \mathrm{m}$. The wet film thickness $h_{\text {wet }}$ was varied via the volume flow. With the help of a syringe pump (Cetoni Nemesys), the volume flow was fed into the die and distributed homogeneously over the entire coating width with the lowest possible pressure drop via distribution cavities. Thereafter, the pressure drop in the die is increased considerably via a narrow slot to enable a lateral distribution of the fluid. The slot is adjusted with a spacer plate, the so-called shim. For the experiments, a slot die with variable shim inlets was used. The shims had a thickness of $500 \mu \mathrm{m}$ with constant coating width $w_{\text {coating }}$ of $50 \mathrm{~mm}$. To modify the volume flow and pressure distribution in the slot die, the shim geometry at the outlet of the die is adjusted (see Fig. 6).

The following process parameters and geometrical parameters for the selected shim configurations were used in the experiments. The parameters are shown in Table 2 .

\section{Characterization methods}

After the coating is applied to the roller, its film profile at the edge of the coating is measured using 2D triangulation lasers (Keyence LJ-V) with a measuring width of $16 \mathrm{~mm}$ each (compare Fig. 5). The 2D data of the coating height and width are then evaluated. For this purpose, at least four measurements of both edge areas are taken. Using two lasers, one for each lateral edge, ensures that the set wet film thickness $h_{\text {wet }}$ of the coating is equal over the entire cross-web profile.

\section{Results and discussion}

Before the experiments started, the process window was calculated to ensure that the operating points were within the area of stable coatings. In Fig. 7, the process window with all experimentally used operating points is shown. The variation of the coating speed at constant wet film thickness $h_{\text {wet }}$ is marked with blue squares (variation coating speed, Fig. 7). The variation of the wet film thickness $h_{\text {wet }}$ at constant coating speed (variation $h_{\text {wet }}$, Fig. 7) is shown with orange diamonds. The experiments with different shim geometries (variation shim geometries, Fig. 7) are marked with green dots. All experiments were performed with a gap of $180 \mu \mathrm{m}$ (gray line). In the process window, the stable coating area is limited by the gap distance $h_{\mathrm{G}}$ as the upper process limit at which barring can occur (gray line) and the lower process limit air entrainment and low-flow limit (dashed line).

With this calculation, it can be expected that stable coatings are possible with the selected operating points. For specific shim geometries, Han et al. showed an influence on the coating window. ${ }^{17}$ The operating point for the variation of the shim geometry (green dot) is selected with a large distance to the process limits. Therefore, the effects that Han et al. found were not observed for the operating points and shim geometries used in this work.

Three different types of height profiles occurred in this work (see Fig. 8). Type 1 is the target profile, which is the motivation of this work. Type 2 is the usual height profile for electrode coatings with comparable materials and type 3 was observed in the variation of shim geometries.

In type 2 , the characteristic edge elevation with $h_{\max }$ is formed for these materials, which transitions into a constant height plateau at the average wet film thickness $h_{\text {wet }}$. The width of the edge elevation is 

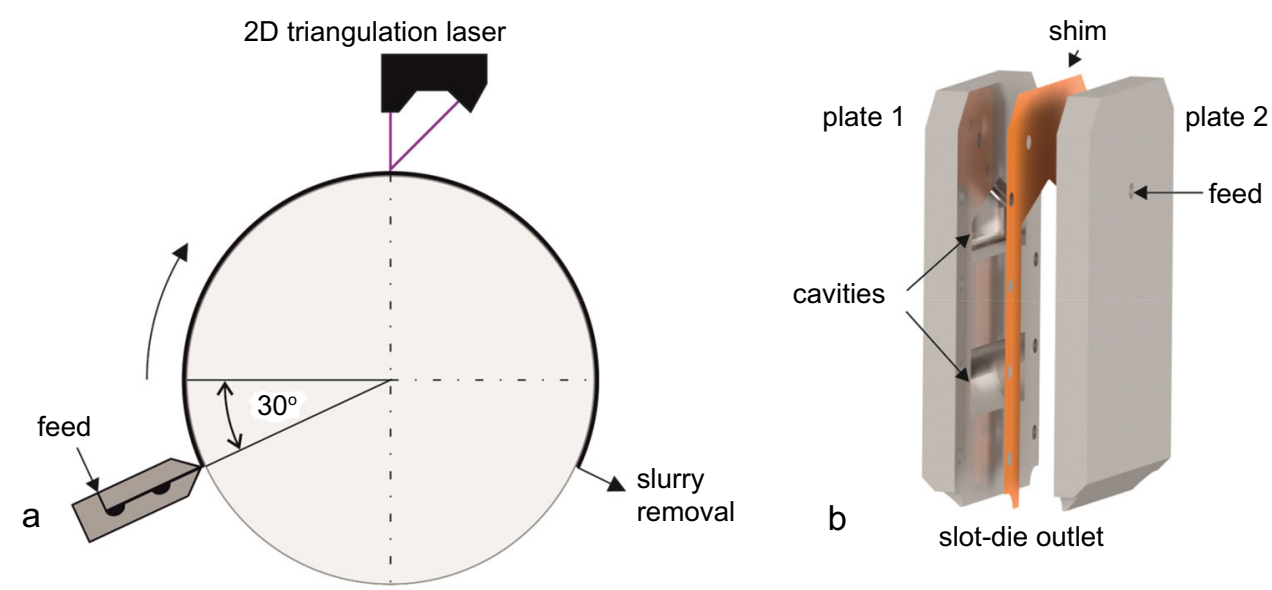

Fig. 5: Schematic illustration of the Development Coater with mounted slot die, high-precision roller, and 2D triangulation laser $(a)^{4}$ and CAD illustration of the used slot die (b)
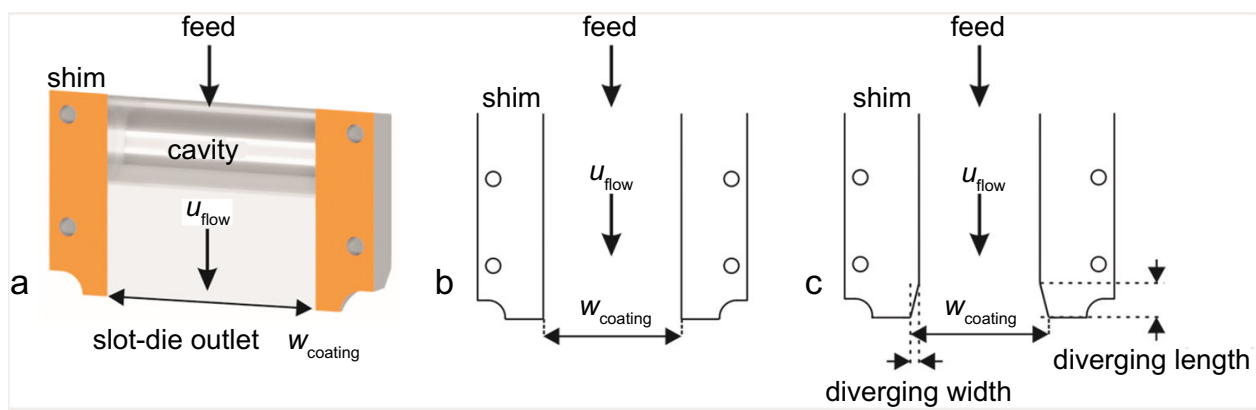

Fig. 6: Schematic illustration of the inside of the slot die (a) and schematic drawing of two types of shim geometries used in the experiments: standard rectangular shim (b) and diverging shim (c)

Table 2: Process parameters and geometrical parameters used in the experiments for the different shim configurations

\begin{tabular}{llcll} 
Figure & Coating speed $/ \mathrm{m} \mathrm{min}^{-1}$ & Dimensionless gap $\mathrm{G}^{*} /-$ & Diverging length $/ \mathrm{mm}$ & Diverging width/mm \\
\hline Figure 6 b & - & 1.00 & 0 (Standard shim) & 0 (Standard shim) \\
Figure 6 c & 5 & 1.10 & 6 & 0.5 \\
Figure 6 c & 10 & 1.15 & 8 & 1.0 \\
Figure 6 c & 20 & 1.20 & 9 & 1.5 \\
Figure 6 c & 50 & 1.40 & 10 & 2.0
\end{tabular}

described with $b_{\text {edge. }}$ In type 1 , the edge superelevation does not exist and the plateau is instantly reached. If an unsuitable shim geometry was used, a characteristic minimum follows in the height profile after the edge superelevation (type 3). For the evaluation of the following experimental results, the height of the edge elevations is shown as dimensionless edge height $H^{*}$.

$H^{*}=\frac{h_{\mathrm{max}}}{h_{\mathrm{wet}}}$

The dimensionless edge height $H^{*}$ is calculated from the quotient of the maximum of the measured edge height $h_{\max }$ and the average wet film thickness $h_{\text {wet }}$ of the plateau towards the coating center. For comparison in dimensionless ratios, the gap is also given in dimensionless form. The dimensionless gap $G^{*}$ is calculated with the gap distance $h_{\mathrm{G}}$ and the wet film thickness $h_{\text {wet }}$

$$
G^{*}=\frac{h_{\mathrm{G}}}{h_{\mathrm{wet}}}
$$




\section{Influence of coating speed}

The influence of the coating speed on the edge elevations is known from the literature. ${ }^{4,9}$ In this work, the influence of the coating speed for the electrode slurry used was verified and analyzed with regard to its effect on the neck-in flow. For these experiments, a standard rectangular shim with an outlet width of $50 \mathrm{~mm}$ was used. The influence of the coating speed on the superelevations was investigated. A gap $h_{\mathrm{G}}=$ $180 \mu \mathrm{m}$ with a dimensionless gap distance $G^{*}$ of 1.15 was set. In Fig. 9, the dimensionless edge height $H^{*}$ is plotted as a function of the coating speed.

The dimensionless edge height $H^{*}$ shows no significant influence of the coating speed between 5 and

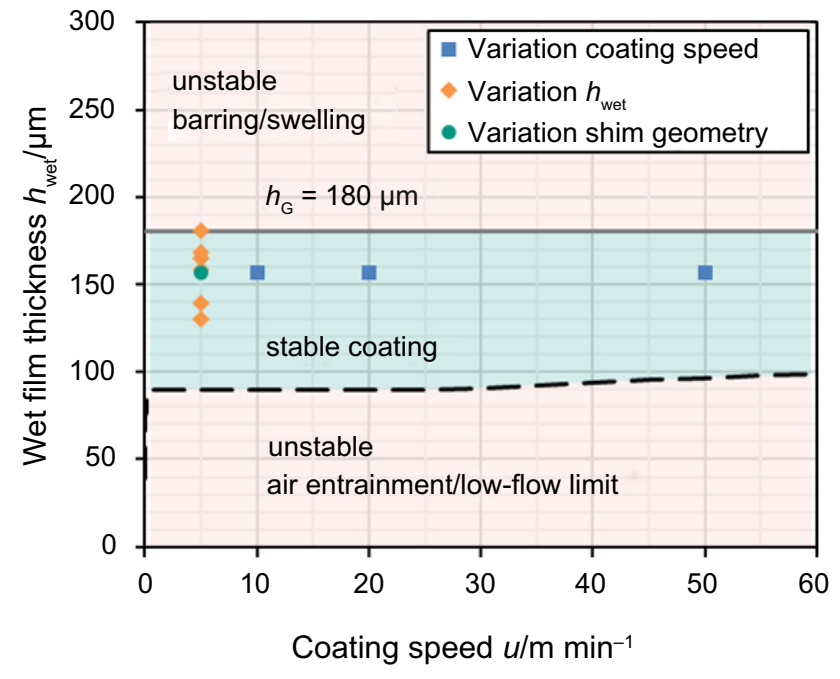

Fig. 7: Process window of all used operating points. Between the gray line and the dashed line, the area for stable coatings, which is limited at the bottom by air entrainment and low-flow limit and at the top by the gap distance $h_{\mathrm{G}}$ is shown
$50 \mathrm{~m} \mathrm{~min}^{-1}$. This observation is in line with the results of Dobroth and Erwin for extrusion coating of cast films. ${ }^{9}$ This observation is also consistent with the results of Schmitt, who described no influence of the coating speed above a gap distance of $100 \mu \mathrm{m}$ in the case of slot die coating of shear-thinning slurries. ${ }^{4}$ When the coating speed is increased for the same nominal wet film thickness $h_{\text {wet }}$, the volume flow and, thus, the average flow speed in the gap must be increased by the same factor. Consequently, the ratio of coating speed $u$ and average speed of the slurry in the gap $\bar{u}_{\text {slurry }}$ remains constant and therefore the conditions for the neck-in flow are the same. Ultimately, this consistency leads to the same dimensionless edge height $H^{*}$ for all coating speeds (compare Fig. 2).

\section{Influence of dimensionless gap}

Beside the coating speed, the dimensionless gap $G^{*}$ is the next important process parameter for slot die coating. To investigate the influence of this parameter on the dimensionless edge height $H^{*}$, the gap was fixed to $180 \mu \mathrm{m}$ and the wet film thickness the coating speed was set to $5 \mathrm{~m} \mathrm{~min}^{-1}$ in the following, due to the low standard deviation of the dimensionless edge height $H^{*}$ and the low material consumption. A standard rectangular shim with an outlet width of $50 \mathrm{~mm}$ was used. Figure 10 shows the dimensionless edge height $H^{*}$ as a function of the dimensionless gap $G^{*}$.

Figure 10 shows that the dimensionless edge height $H^{*}$ increases linearly with increasing dimensionless gap $G^{*}$ with the slope of 0.15 . This observation is in line with the results of Dobroth and Erwin for extrusion coating $^{9}$ and also consistent with the results of Schmitt for slot die coating of shear-thinning electrode slurries. ${ }^{4}$ By increasing the dimensionless gap $G^{*}$, the wet film thickness $h_{\text {wet }}$ decreases in relation to the gap $h_{\mathrm{G}}$. This adjustment is accompanied by an increased neckin flow due to an increased ratio of coating speed and

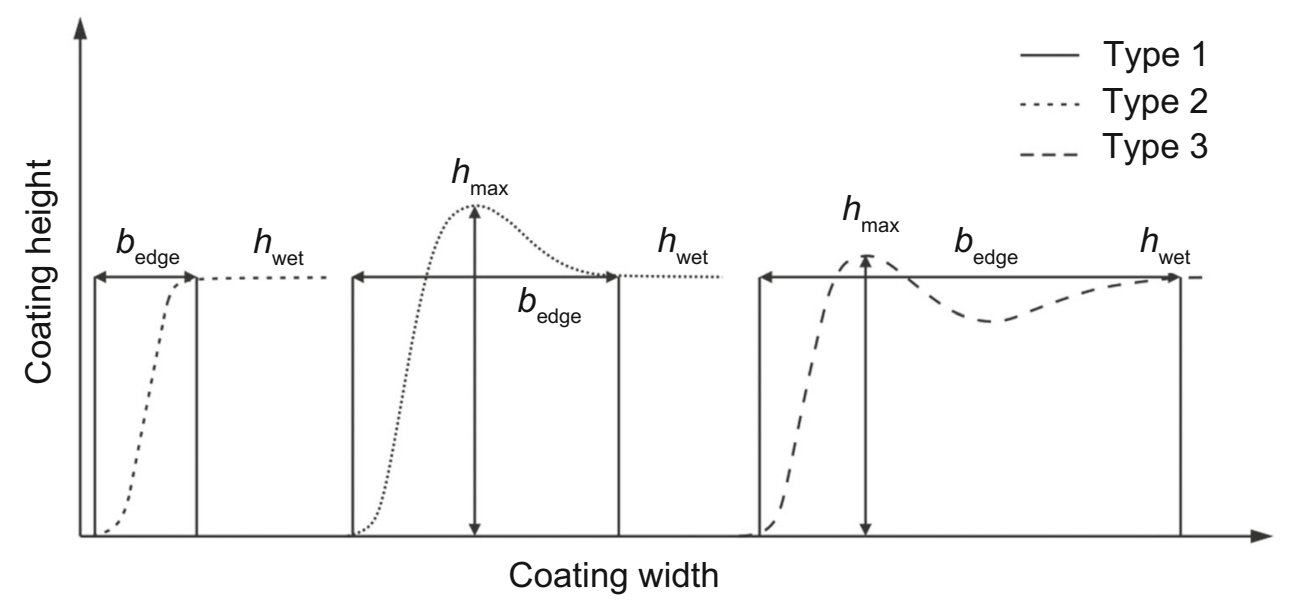

Fig. 8: Three types of wet film thickness $h_{\text {wet }}$ cross-web profiles observed in this work 


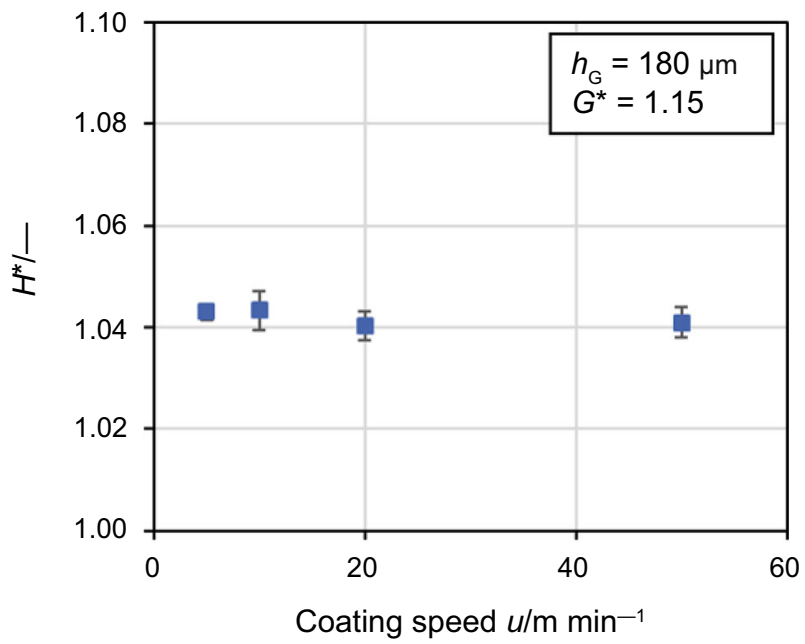

Fig. 9: Dimensionless edge height $H^{*}$ as a function of the coating speed with $h_{\mathrm{G}}=180 \mu \mathrm{m}$ and $G^{*}=1.15$ for a standard rectangular shim geometry

average speed of the slurry in the gap. Due to the higher local flow rate in cross-web direction, the neckin flow increases due to material entry from the lateral menisci toward the center of the coating (see Fig. 2). $4,7,9$

\section{Influence of shim geometries}

As mentioned before, in the experiments, three types of wet film thickness profiles were observed. The types resulted from the variation of the shim geometries. In Fig. 11, experimental examples for each different type are shown.

For coatings with the diverging shim geometry and an optimal adjustment of diverging length and diverging width, the target profile type 1 was observed. When varying the shim geometries, type 2 profiles were found for small changes of the diverging shim geometry compared to the standard rectangular shim. If the diverging width was set too large, it negatively influenced the flow at the die outlet and, thus, the neck-in flow and type 3 profiles were observed. For coatings with the standard rectangular shim mounted in the slot die, wet film thickness profiles of types 1 and 2 were observed. By decreasing $G^{*}$ as described above, the edge elevations could be reduced, resulting in the target profile type 1 .

As described in the literature and shown with experimental results before, it is possible to reduce the edge superelevations by adjusting the process parameters. ${ }^{4,9}$ As mentioned above, especially an adjustment of the gap $h_{\mathrm{G}}$ can have negative effects on the process stability. Therefore, the aim of this work is to modify the edge elevations with a method at constant process parameters. The influence of the shim geometry on the dimensionless edge height $H^{*}$ was investigated with a variation of diverging length and

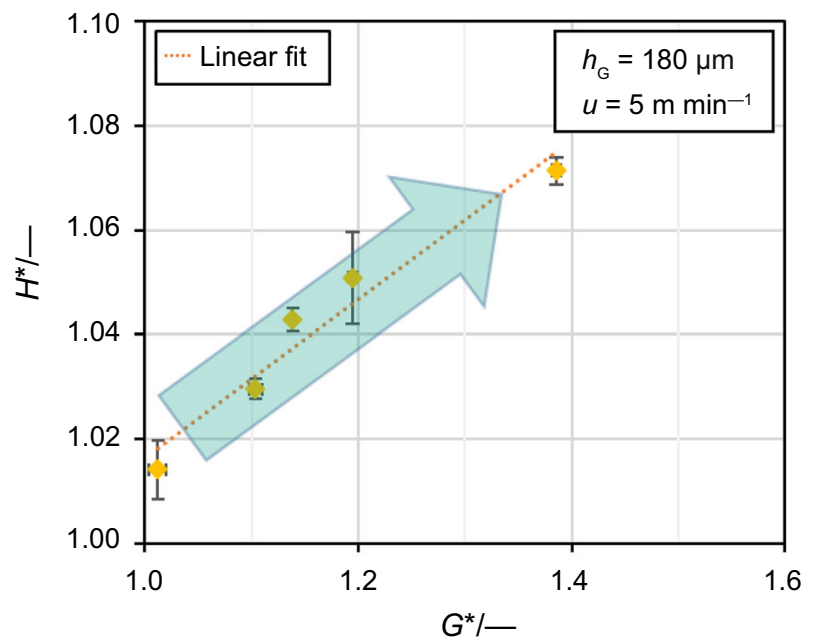

Fig. 10: Dimensionless edge height $\boldsymbol{H}^{*}$ as a function of the dimensionless gap $G^{*}$ at set $h_{\mathrm{G}}=180 \mu \mathrm{m}$ and a coating speed of $5 \mathrm{~m} \mathrm{~min}^{-1}$ for a standard rectangular shim. The results can be fitted as a linear fit to equation $\boldsymbol{H}^{*}=0.15 G^{*}+$ 0.86

diverging width. The experiments were performed at a constant gap distance $h_{\mathrm{G}}$ of $180 \mu \mathrm{m}$ and a coating speed of $5 \mathrm{~m} \mathrm{~min}^{-1}$. The dimensionless gap $G^{*}$ was set to $G^{*}=1.15$. First, the diverging length was varied starting from a standard shim (blue square). In the experiments, the diverging length was increased with different shims within the die. The results of the dimensionless edge height $H^{*}$ (top) and edge width $b_{\text {edge }}$ (bottom) are shown in Fig. 12 as a function of the diverging length. The results for the variation of the diverging length with a diverging width of $1.5 \mathrm{~mm}$ are shown in green dots.

The results show that if the diverging length of the shim is opened from the standard rectangular shim to a diverging shim, the dimensionless edge height $H^{*}$ is nearly constant in terms of standard deviation up to a diverging length of $8 \mathrm{~mm}$. The edge width $b_{\text {edge }}$ is also nearly constant up to diverging length of $9 \mathrm{~mm}$. The influence of the diverging length on the dimensionless edge height $H^{*}$ and edge width $b_{\text {edge }}$ is not significant. For these results, a height profile of type 2 is observed. If the diverging length is increased to $10 \mathrm{~mm}$, both the dimensionless edge height $H^{*}$ and the edge width $b_{\text {edge }}$ decrease. The influence of the diverging length at this point is sufficiently large to decrease the local flow at the area of the coating edge inside the die. This ensures that the ratio of web speed and average flow velocity and thus, the neck-in flow increases. The locally lower flow at the region of the coating edge, however, shows a greater influence than the increased neck-in flow. Therefore, the dimensionless edge height $H^{*}$ decreases. At $10 \mathrm{~mm}$, the edge elevation has an absolute value of approximately $1.3 \mu \mathrm{m}$ (difference of maximum $h_{\max }$ edge height and average wet film thickness $h_{\text {wet }}$ ). The evaluation results in an edge width $b_{\text {edge }}$ of approximately $1.8 \mathrm{~mm}$ at a diverging length of 


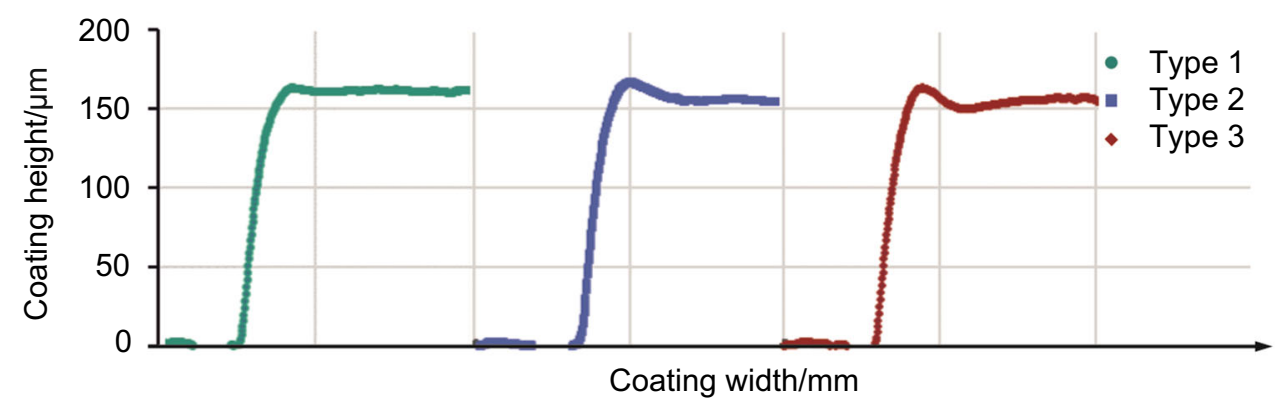

Fig. 11: Different types of wet film thickness profiles observed in the experiments (experimental results)
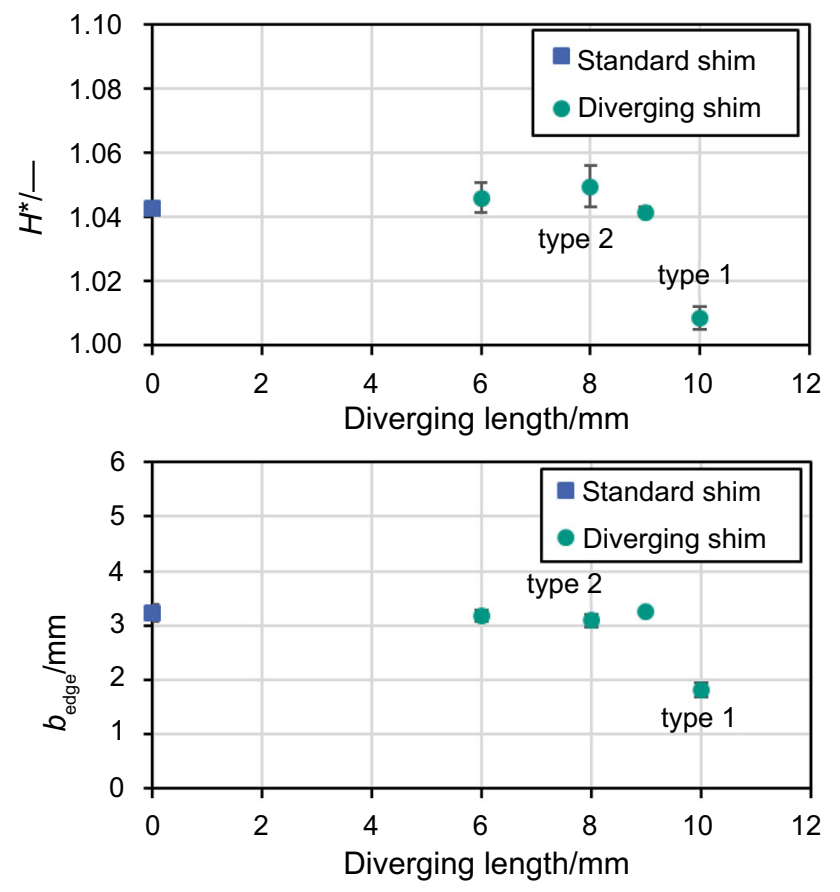

Fig. 12: Results of dimensionless edge height $\boldsymbol{H}^{*}$ (top) and edge width $b_{\text {edge }}$ (bottom) as a function of the diverging length. Standard rectangular shim (blue square) and different diverging shims (green dots) are compared for a coating speed of $5 \mathrm{~m} \mathrm{~min}^{-1}$, a gap of $180 \mu \mathrm{m}$ and $G^{*}=1.15$

$10 \mathrm{~mm}$, which is negligible for the falling height profile and the small height of the superelevation (see height profile type 1). These results show that the edge elevations are influenced by the diverging length. The increase of the diverging length from 8 to $10 \mathrm{~mm}$ leads to a significant change in the flow distribution at the die outlet. Thus, the ratio of web speed and average flow velocity and the neck-in flow increases. However, the influence of the locally lower flow at the region of the coating edges leads to a decreased dimensionless edge height. This observation can be also compared with the results of Han et al. ${ }^{17}$ A reduction of the pressure and, therefore, the local flow at the area at the coating edge due to a higher diverging length results in a reduction of edge elevation.
So far the influence of the diverging length has been investigated. Based on these results, the optimum diverging width needs to be determined. In the following, the diverging width was varied starting from a standard rectangular shim (blue square). In the experiments, the diverging width was increased with different shims within the die. The diverging length was set to $10 \mathrm{~mm}$. The experiments were carried out at the same conditions as before. The results of the dimensionless edge height $H^{*}$ (top) and edge width $b_{\text {edge }}$ (bottom) are shown in Fig. 13 as a function of the diverging width. The results for the variation of the diverging shim are shown as green dots.

If the diverging width of the shim increases, the dimensionless height $H^{*}$ and edge width $b_{\text {edge }}$ are nearly constant up to a diverging width of $1 \mathrm{~mm}$. Up to this value, type 2 height profiles are observed. If the diverging width increases to $1.5 \mathrm{~mm}$, both the dimensionless edge height $H^{*}$ and the edge width $b_{\text {edge }}$ decrease. At this point, the influence of the diverging width on the local flow is high enough to decrease the dimensionless edge height $H^{*}$ and edge width $b_{\text {edge }}$. This observation is consistent with Han et al.; they also found an influence of the diverging shim on the local volume flow inside the die. ${ }^{17}$ At $1.5 \mathrm{~mm}$, the edge elevation has an absolute value of approximately $1.3 \mu \mathrm{m}$ like the result for the variation of the diverging length. The evaluation results in an edge width $b_{\text {edge }}$ of approximately $1.8 \mathrm{~mm}$ at a diverging width of $1.5 \mathrm{~mm}$. Analogous to increasing the diverging length, increasing the diverging width also changes the local volume flow at the die outlet. The ratio of web speed and average flow velocity increases and thus, the neck-in flow, but the impact of the decreasing local flow at the region of the coating edges is stronger, which leads to a reduction of the edge elevation. If the diverging width is increased to $2 \mathrm{~mm}$, both the dimensionless edge height $H^{*}$ and the edge width $b_{\text {edge }}$ increase strongly. The absolute value of $9.3 \mu \mathrm{m}$ is reached for the difference of maximum edge height $h_{\max }$ and average wet film thickness $h_{\text {wet }}$ and $4.1 \mathrm{~mm}$ for the edge width $b_{\text {edge. }}$. If the diverging width is large and, thus, the local flow low, the result will be a local minimum in the height profile (type 3 ). Due to the local minimum in the height profile, the edge width $b_{\text {edge }}$ increases and wet film thickness $h_{\text {wet }}$ reaches the plateau significantly 

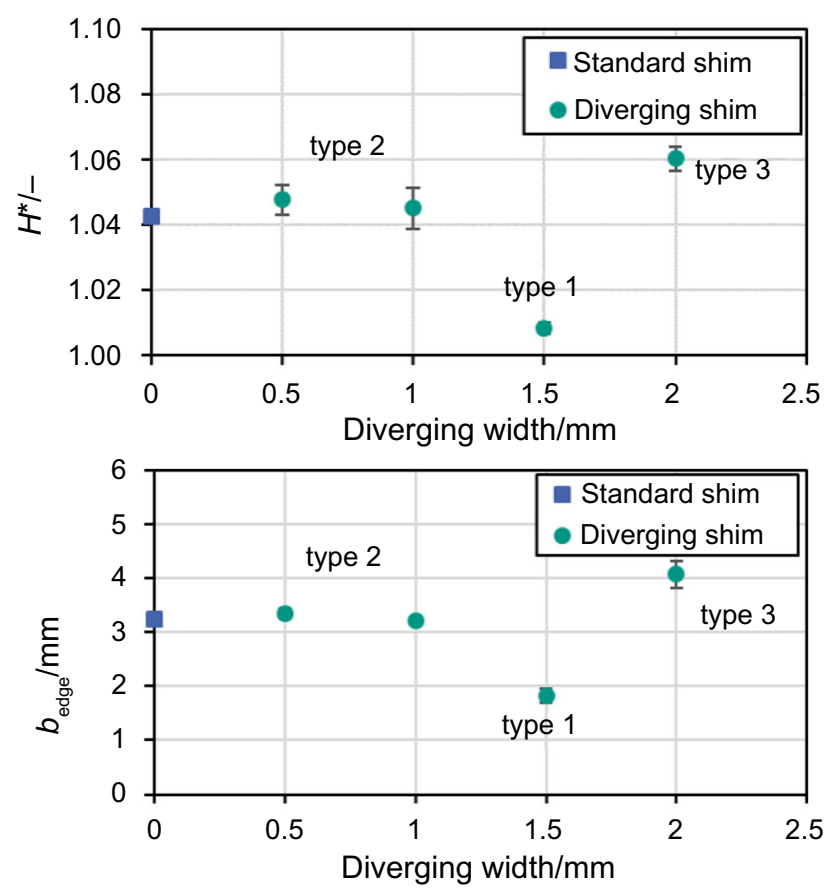

Fig. 13: Results of dimensionless edge height $\boldsymbol{H}^{*}$ (top) and edge width $b_{\text {edge }}$ (bottom) as a function of the diverging width. Standard rectangular shim (blue square) and different diverging shims (green dots) are compared for a coating speed of $5 \mathrm{~m} \mathrm{~min}^{-1}$, a gap of $180 \mu \mathrm{m}$ and $G^{*}=1.15$

further from the edge which reduces the average wet film thickness in the evaluation of the results. This minimum leads also to a higher dimensionless edge height $H^{*}$. Concludingly, for the operation point with a diverging length of $10 \mathrm{~mm}$ and a diverging width of $1.5 \mathrm{~mm}$, there is no significant edge elevation observed (type 1).

\section{Conclusions}

In today's electrode production, elevations at the lateral edges occur in the coating step. These superelevations lead to defects in subsequent process steps and, therefore, to reject in the process. In order to reduce edge elevations, a method with constant process parameters was investigated. For this purpose, an electrode slurry was coated with a slot die onto a high-precision roller and the wet film height profile was then recorded using a $2 \mathrm{D}$ triangulation laser. Process parameters, such as dimensionless gap $G^{*}$ and coating speed as well as the shim geometries inside the slot die, were varied to investigate different influences on the formation of edge elevations.

It was shown that the edge elevations can be eliminated by a suitable selection of the shim geometry within the slot die at constant process parameters. With the adjustment of the diverging length to $10 \mathrm{~mm}$ and the diverging width to $1.5 \mathrm{~mm}$, no significant edge elevations were observed at a coating speed of $5 \mathrm{~m} \mathrm{~min}^{-1}$ with a gap distance $h_{\mathrm{G}}$ of $180 \mu \mathrm{m}$ at $G^{*}$ of 1.15. A challenge, however, remains to find the appropriate geometry, which is very time consuming. In addition, the influence of the process parameters on the formation of superelevations for the material system used in this work, as already shown by Schmitt, was verified. ${ }^{4}$ On the one hand, the reduction of $G^{*}$ can lead to an elimination of the superelevations. By choosing $G^{*}=1$ at a coating speed of $5 \mathrm{~m} \mathrm{~min}^{-1}$ and a gap distance $h_{\mathrm{G}}$ of $180 \mu \mathrm{m}$, the edge elevations could also be almost eliminated. However, according to the literature, the use of $G^{*}<1$ carries the risk of producing inhomogeneous coatings through barring. On the other hand, the variation of the process speed has no significant influence on the formation of superelevations. This independency is especially advantageous for the ramp-up of the process speed and the other parameters and shim geometries can be maintained.

Finally, it is necessary to clarify how the influence of the process parameters on the edge elevations is with adapted shim geometries. Furthermore, the process limits for variations of the shim geometries should be investigated. As already observed by $\mathrm{Han}$ et al. for Newtonian fluids, it is also to be expected that for shear-thinning media, due to the local reduction of the outlet flow, the edge will show stability limiting effects at high coating speeds or small wet film thicknesses $h_{\text {wet }}$. It can also be expected that the suitable shim geometry will depend on the material and that an investigation of the influence of viscosity will be particularly useful.

Acknowledgments The authors would like to acknowledge financial support of the Federal Ministry of Education and Research (BMBF) via the ProZell cluster-project "HiStructures" (Grant Number: 03XP0243C). The authors would like to thank all involved mechanics at the institute and Julian Klemens for particular contributions to this work.

Funding Open Access funding enabled and organized by Projekt DEAL.

Open Access This article is licensed under a Creative Commons Attribution 4.0 International License, which permits use, sharing, adaptation, distribution and reproduction in any medium or format, as long as you give appropriate credit to the original author(s) and the source, provide a link to the Creative Commons licence, and indicate if changes were made. The images or other third party material in this article are included in the article's Creative Commons licence, unless indicated otherwise in a credit line to the material. If material is not included in the article's Creative Commons licence and your intended use is not permitted by statutory regulation or exceeds the permitted use, you will need to obtain permission directly from 
the copyright holder. To view a copy of this licence, visit http://creativecommons.org/licenses/by/4.0/.

\section{References}

1. Korthauer, R (ed) Handbuch Lithium-Ionen-Batterien. Springer, Berlin Heidelberg, Berlin, Heidelberg, s.l. (2013)

2. Kaiser, J, Wenzel, V, Nirschl, H, Bitsch, B, Willenbacher, N, Baunach, M, Schmitt, M, Jaiser, S, Scharfer, P, Schabel, W, "Prozess- und Produktentwicklung von Elektroden für LiIonen-Zellen." Chemie Ingenieur Technik, 86 (5) 695-706 (2014)

3. Eser, JC, Wirsching, T, Weidler, PG, Altvater, A, Börnhorst, T, Kumberg, J, Schöne, G, Müller, M, Scharfer, P, Schabel, $\mathrm{W}$, "Moisture Adsorption Behavior in Anodes for Li-Ion Batteries." Energy Technol., 8 (2) 1801162 (2020)

4. Schmitt, M, Slot Die Coating of Lithium-ion Battery Electrodes (2016)

5. Kim, S, Lee, J, Lee, C, "Computational Fluid Dynamics Model for Thickness and Uniformity Prediction of Coating Layer in Slot-Die Process." Int. J. Adv. Manuf. Technol., 104 (5-8) 2991-2997 (2019)

6. Kistler, SF, Schweizer, PM, Liquid Film Coating. Scientific Principles and Their Technological Implications. Springer, Dordrecht (2012)

7. Gutoff, EB, Cohen, ED, Kheboian, GI, Coating and Drying Defects. Troubleshooting Operating Problems. Wiley, Hoboken (2006)

8. Günther, T, Schreiner, D, Metkar, A, Meyer, C, Kwade, A, Reinhart, G, "Classification of Calendering-Induced Electrode Defects and Their Influence on Subsequent Processes of Lithium-Ion Battery Production." Energy Technol., 8 (2) 1900026 (2020)
9. Dobroth, T, Erwin, L, "Causes of Edge Beads in Cast Films." Polym. Eng. Sci., 26 (7) $462-467$ (1986)

10. Schmitt, M, Scharfer, P, Schabel, W, "Slot Die Coating of Lithium-Ion Battery Electrodes: Investigations on Edge Effect Issues for Stripe and Pattern Coatings." J. Coat. Technol. Res., 11 (1) 57-63 (2014)

11. Schmitt, M, Baunach, M, Wengeler, L, Peters, K, Junges, P, Scharfer, P, Schabel, W, "Slot-Die Processing of Lithium-Ion Battery Electrodes-Coating Window Characterization." Chem. Eng. Process. Process Intensif., 68 32-37 (2013)

12. Schmitt, M, Diehm, R, Scharfer, P, Schabel, W, "An Experimental and Analytical Study on Intermittent Slot Die Coating of Viscoelastic Battery Slurries." J. Coat. Technol. Res., 12 (5) 927-938 (2015)

13. Diehm, R, Weinmann, H, Kumberg, J, Schmitt, M, Fleischer, J, Scharfer, P, Schabel, W, "Edge Formation in High-Speed Intermittent Slot-Die Coating of Disruptively Stacked Thick Battery Electrodes." Energy Technol., 51900137 (2019)

14. Tsuda, T, "Coating Flows of Power-Law Non-Newtonian Fluids in Slot Coating." J. Soc. Rheol. Jpn., 38 (4/5) 223-230 (2010)

15. Ruschak, KJ, "Limiting Flow in a Pre-metered Coating Device.” Chem. Eng. Sci., 31 (11) 1057-1060 (1976)

16. Carvalho, MS, Kheshgi, HS, "Low-Flow Limit in Slot Coating: Theory and Experiments." AIChE J., 46 (10) 1907-1917 (2000)

17. Han, GH, Lee, SH, Ahn, W-G, Nam, J, Jung, HW, "Effect of Shim Configuration on Flow Dynamics and Operability Windows in Stripe Slot Coating Process." J. Coat. Technol. Res., 11 (1) 19-29 (2014)

Publisher's Note Springer Nature remains neutral with regard to jurisdictional claims in published maps and institutional affiliations. 